\title{
Pairing fluctuation effects on the single-particle spectra for the superconducting state
}

\author{
P. Pieri, L. Pisani, and G.C. Strinati \\ Dipartimento di Fisica, UdR INFM, Università di Camerino, I-62032 Camerino, Italy
}

(November 4, 2018)

\begin{abstract}
Single-particle spectra are calculated in the superconducting state for a fermionic system with an attractive interaction, as functions of temperature and coupling strength from weak to strong. The fermionic system is described by a single-particle self-energy that includes pairing-fluctuation effects in the superconducting state. The theory reduces to the ordinary BCS approximation in weak coupling and to the Bogoliubov approximation for the composite bosons in strong coupling. Several features of the single-particle spectral function are shown to compare favorably with experimental data for cuprate superconductors.
\end{abstract}

PACS numbers: 03.75.Ss, 03.75.Hh, 05.30.Jp

Information on the single-particle spectral function, that is obtained from ARPES ${ }^{1}$ and tunneling data ${ }^{2}$ for cuprate superconductors, can shed light on the characteristic features of the superconducting state as well as on its connection with the unconventional normal state above the critical temperature $T_{c}$.

Most prominent among these features are the continuous evolution of a broad pseudogap structure from above to below $T_{c},{ }^{1,2}$ the emergence of a coherent peak below $T_{c}$ that combines with the pseudogap structure to yield a characteristic peak-dip-hump profile ${ }^{1}$ (for which two distinct energy scales can be identified), and the peculiar dependence of the frequency position and weight of the coherent peak on temperature and doping. ${ }^{1}$ Generally speaking, features of the standard BCS theory are recovered for overdoped samples, while non-BCS behaviors occur for optimally-doped and underdoped samples.

The origin of the peak-dip-hump profile has especially been the subject of controversy, being attributed either to "extrinsic" effects like the bilayer splitting ${ }^{3}$ or to "intrinsic" effects. The latter can be identified over and above the extrinsic effects, and are believed to originate from strong (many-body) interactions in the system ${ }^{4}$.

Quite independently from the microscopic origin of the fermionic attraction giving rise to superconductivity, its strength is believed to be stronger in optimallydoped and underdoped than in overdoped samples ${ }^{5}$, consistently with the above findings. This implies that pairing fluctuations should definitely be taken into account, irrespective of other effects (such as the bilayer splitting and/or additional many-body effects associated with specific pairing mechanisms ${ }^{6}$ ).

In this paper, we assess the role of pairing fluctuations for the single-particle spectral function in the superconducting state on rather general grounds, by identifying a single-particle self-energy that describes fluctuating Cooper pairs in weak coupling and non-condensed composite bosons in strong coupling. (The latter form as bound-fermion pairs in the strong-coupling limit of the fermionic attraction.) To this end, we consider fermions mutually interacting via an attractive contact potential in a 3D continuum, without taking into account lattice effects nor the explicit physical mechanism responsible for the attraction. Qualitative comparison with experimental data will thus rest on identifying the increasing coupling strength in this model with the increasing potential strength for decreasing doping level in the phase diagram of cuprate superconductors. Although the effective model here considered is oversimplified for a full description of cuprates, the physical questions we are addressing are sufficiently general that this minimal model will prove sufficient to capture the main experimental features.

Our main results for the single-particle spectral function (to be compared with the experimental findings) are summarized as follows:

(i) A broad pseudogap structure and a coherent peak are simultaneously present in a wide range of coupling and temperature below $T_{c}$, giving rise to a peak-dip-hump profile. Two distinct energy scales (the positions $\Delta_{\mathrm{pg}}$ of the broad pseudogap structure and $\Delta_{\mathrm{m}}$ of the coherent peak) can then be extracted from the spectra at given temperature and coupling, as seen experimentally ${ }^{1}$.

(ii) At a given (notably, intermediate) coupling, BCS-like features coexist with non-BCS behaviors. The position $\Delta_{\mathrm{m}}$ and weight $z$ of the coherent peak versus wave vector follow a BCS-like dependence, as found experimentally $^{7,8}$. At the same time, the weight $z$ of the coherent peak has a strong temperature dependence, in agreement with experiments ${ }^{9,10}$ but in contrast with BCS theory.

(iii) At low temperature, the weight $z$ of the coherent peak has a strong dependence on coupling, decreasing monotonically from weak to strong coupling (as seen experimentally in cuprates for decreasing doping $\left.{ }^{9,10}\right)$. At the same time, the position $\Delta_{\mathrm{m}}$ of the coherent peak increases monotonically with coupling ${ }^{9,10}$.

(iv) The positions $\Delta_{\mathrm{pg}}$ of the broad pseudogap structure at $T_{c}$ and $\Delta_{\mathrm{m}}$ of the coherent peak near zero temperature 
cross each other as function of coupling about intermediate coupling. This feature is also seen experimentally by intrinsic tunneling experiments at different dopings ${ }^{11}$ (although these data are subject to controversy ${ }^{12}$ ).

Pairing fluctuations are taken into account in our theory by considering, besides the off-diagonal BCS-like selfenergy, the diagonal $t$-matrix self-energy suitably extended to the superconducting state (we use the Nambu formalism throughout). The $t$-matrix self-energy has been widely used to include pairing fluctuations in the normal state ${ }^{13}$, and specifically to account for pseudogap features in the single-particle spectral function ${ }^{14}$. In our theory, the diagonal $t$-matrix self-energy (that survives above $T_{c}$ ) will essentially be responsible for the presence of the broad pseudogap structure below $T_{c}$. The off-diagonal BCS-like self-energy will instead give rise to the simultaneous emergence of the coherent peak. In addition, our theory recovers the Bogoliubov approximation for the composite bosons in strong coupling. ${ }^{15}$

The diagonal $t$-matrix self-energy reads

$$
\Sigma_{11}(k)=-\int \frac{d \mathbf{q}}{(2 \pi)^{3}} \frac{1}{\beta} \sum_{\Omega_{\nu}} \Gamma_{11}(q) \mathcal{G}_{11}(q-k),
$$

while the off-diagonal self-energy has the BCS-like form $\Sigma_{12}(k)=-\Delta$ in terms of the superconducting order parameter $\Delta$. The pairing-fluctuation propagator $\Gamma_{11}$ in the broken-symmetry phase entering Eq. (1) is given by:

$$
\Gamma_{11}(q)=\frac{\chi_{11}(-q)}{\chi_{11}(q) \chi_{11}(-q)-\chi_{12}(q)^{2}}
$$

with

$$
\begin{aligned}
&-\chi_{11}(q)=\frac{m}{4 \pi a_{F}}+\int \frac{d \mathbf{p}}{(2 \pi)^{3}}\left[\frac{1}{\beta} \sum_{\omega_{n}} \mathcal{G}_{11}(p+q) \mathcal{G}_{11}(-p)\right. \\
&\left.-\frac{m}{\mathbf{p}^{2}}\right] \\
& \chi_{12}(q)=\int \frac{d \mathbf{p}}{(2 \pi)^{3}} \frac{1}{\beta} \sum_{\omega_{n}} \mathcal{G}_{12}(p+q) \mathcal{G}_{21}(-p)
\end{aligned}
$$

In the above expressions, $q=\left(\mathbf{q}, \Omega_{\nu}\right), k=\left(\mathbf{k}, \omega_{l}\right)$, and $p=\left(\mathbf{p}, \omega_{n}\right)\left(\mathbf{q}, \mathbf{k}\right.$, and $\mathbf{p}$ being wave vectors, $\Omega_{\nu}$ a bosonic Matsubara frequency, and $\omega_{l}$ and $\omega_{n}$ fermionic Matsubara frequencies), $m$ is the fermionic mass, $\beta$ the inverse temperature, and $\mathcal{G}_{i j}(i, j=1,2)$ have the form of the BCS single-particle Green's functions in Nambu notation.

For given temperature and strength of the pointcontact interaction, the chemical potential $\mu$ and the order parameter $\Delta$ are obtained by solving the coupled particle number and gap equations:

$$
\begin{aligned}
n & =2 \int \frac{d \mathbf{k}}{(2 \pi)^{3}} \frac{1}{\beta} \sum_{\omega_{l}} e^{i \omega_{l} \eta} G_{11}\left(\mathbf{k}, \omega_{l}\right) \\
-\frac{m}{4 \pi a_{F}} & =\int \frac{d \mathbf{k}}{(2 \pi)^{3}}\left[\frac{\tanh (\beta E(\mathbf{k}) / 2)}{2 E(\mathbf{k})}-\frac{m}{\mathbf{k}^{2}}\right]
\end{aligned}
$$

where $\eta=0^{+}$and $E(\mathbf{k})=\left[\xi(\mathbf{k})^{2}+\Delta^{2}\right]^{1 / 2}$ with $\xi(\mathbf{k})=$ $\mathbf{k}^{2} /(2 m)-\mu$. The scattering length $a_{F}$ of the (fermionic) two-body problem has been introduced to eliminate the ultraviolet divergences originating from the use of a point-contact interaction. The dimensionless parameter $\left(k_{F} a_{F}\right)^{-1}$ (where $k_{F}$ is the Fermi wave vector related to the density $n$ via $\left.k_{F}=\left(3 \pi^{2} n\right)^{1 / 3}\right)$ characterizes the fermionic coupling strength and ranges formally from $-\infty$ to $+\infty$. [Correspondingly, the Fermi energy $\varepsilon_{F}$ equals $k_{F}^{2} /(2 m)$.] In practice, the crossover from the weak- to strong-coupling regimes occurs in the limited range $-1 \lesssim\left(k_{F} a_{F}\right)^{-1} \lesssim+1$, which will be mostly explored in what follows. The dressed normal (diagonal) Green's function $G_{11}$ in Eq. (5) is obtained by matrix inversion of the Dyson's equation in Nambu formalism with the above self-energies and is given by:

$$
\begin{aligned}
G_{11}\left(\mathbf{k}, \omega_{l}\right) & =\left[i \omega_{l}-\xi(\mathbf{k})-\Sigma_{11}\left(\mathbf{k}, \omega_{l}\right)\right. \\
& \left.-\frac{\Delta^{2}}{i \omega_{l}+\xi(\mathbf{k})+\Sigma_{11}\left(\mathbf{k},-\omega_{l}\right)}\right]^{-1}
\end{aligned}
$$

Note that, while the number equation (5) contains the dressed normal (diagonal) Green's function, the gap equation (6) is obtained from the anomalous (offdiagonal) BCS Green's function and thus its form is not modified with respect to the BCS theory. This ensures that the pairing-fluctuation propagator (2) remains gapless for all temperatures (below $T_{c}$ ) and couplings. [The value of $T_{c}$ is obtained from Eqs. (5) and (6) by setting $\Delta=0$ identically.] The numerical values of the chemical potential $\mu$ and order parameter $\Delta$ at given temperature and coupling differ, however, from those obtained by BCS theory due to the different structure of the number equation. It can be verified ${ }^{15}$ from Eqs. (2)-(6) that the Bogoliubov approximation for the composite bosons is recovered in the strong-coupling $(\beta \mu \rightarrow-\infty$ and $\Delta \ll|\mu|)$ limit. This represents a notable achievement of our theory.

The use of bare BCS single-particle Green's functions in the self-energy (1) further enables us to perform the analytic continuation to real frequency $\omega$ in a closed form, as to avoid numerical extrapolation procedures. To this end, and similarly to what was done in Ref. 14 for the normal phase, we express the pairing-fluctuation propagator $\Gamma_{11}$ by its spectral representation:

$$
\Gamma_{11}\left(\mathbf{q}, \Omega_{\nu}\right)=-\frac{1}{\pi} \int_{-\infty}^{+\infty} d \omega^{\prime} \frac{\operatorname{Im} \Gamma_{11}\left(\mathbf{q}, \omega^{\prime}\right)}{i \Omega_{\nu}-\omega^{\prime}}
$$

Here $\operatorname{Im} \Gamma_{11}(\mathbf{q}, \omega)$ is defined as the imaginary part of $\Gamma_{11}\left(\mathbf{q}, i \Omega_{\nu} \rightarrow \omega+i \eta\right)$. This quantity is, in turn, obtained from the expressions (2)-(4), with the replacement $i \Omega_{\nu} \rightarrow \omega+i \eta$ made after the sum over the internal (Matsubara) frequency has been performed. In this way, the imaginary part of the retarded self-energy $\Sigma_{11}^{R}$ is: 


$$
\begin{aligned}
\operatorname{Im} \Sigma_{11}^{R}(\mathbf{k}, \omega) & =-\int \frac{d \mathbf{q}}{(2 \pi)^{3}}\left\{u_{\mathbf{q}-\mathbf{k}}^{2} \operatorname{Im} \Gamma_{11}(\mathbf{q}, \omega+E(\mathbf{q}-\mathbf{k}))\right. \\
& \times[f(E(\mathbf{q}-\mathbf{k}))+b(\omega+E(\mathbf{q}-\mathbf{k}))] \\
& +v_{\mathbf{q}-\mathbf{k}}^{2} \operatorname{Im} \Gamma_{11}(\mathbf{q}, \omega-E(\mathbf{q}-\mathbf{k})) \\
& \times[f(-E(\mathbf{q}-\mathbf{k}))+b(\omega-E(\mathbf{q}-\mathbf{k}))]\} .
\end{aligned}
$$

Here, $f(x)=[\exp (\beta x)+1]^{-1}$ and $b(x)=[\exp (\beta x)-1]^{-1}$ are the Fermi and Bose distributions, while $u_{\mathbf{k}}^{2}$ and $v_{\mathbf{k}}^{2}$ are the usual BCS coherence factors. The real part of $\Sigma_{11}^{R}$ is then obtained via a Kramers-Kronig transform. The retarded single-particle Green's function $G_{11}^{R}$ results from these functions, in terms of which the single-particle spectral function $A(\mathbf{k}, \omega)=-\frac{1}{\pi} \operatorname{Im} G_{11}^{R}(\mathbf{k}, \omega)$ of interest is eventually obtained as a function of $\omega$ for any given $\mathbf{k} .{ }^{16}$

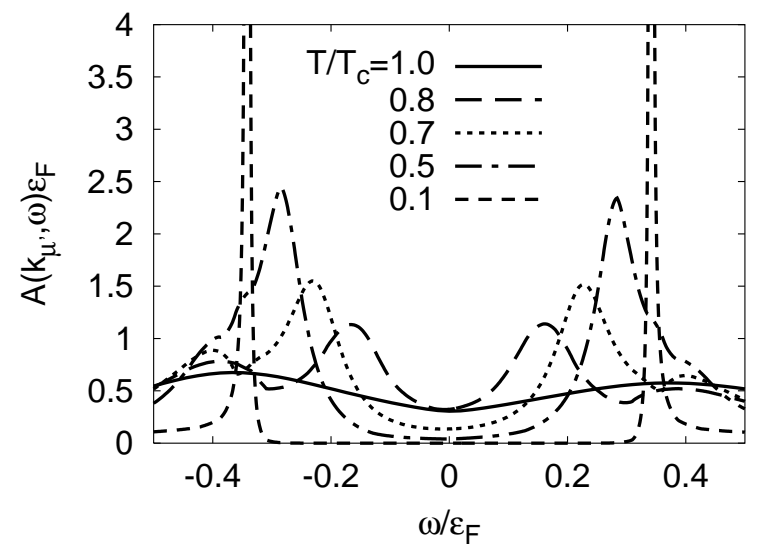

FIG. 1. Single-particle spectral function vs frequency (in units of $\left.\varepsilon_{F}\right)$ at different temperatures, for $|\mathbf{k}|=k_{\mu^{\prime}}$ and $\left(k_{F} a_{F}\right)^{-1}=-0.45$.

Figure 1 shows the evolution with temperature of the single-particle spectral function for an intermediate coupling $\left(\left(k_{F} a_{F}\right)^{-1}=-0.45\right)$. [For this coupling, the ratio of the pair-breaking temperature $T^{*}$ to the critical temperature $T_{c}$ is about 1.25, as obtained in Ref. 14.] Focusing specifically on the features at negative $\omega$, note how the (sharp) coherent peak grows from the (broad) pseudogap structure already present at $T_{c}$. The coherent peak becomes sharper upon lowering the temperature and gains weigth at the expenses of the pseudogap structure, giving rise to a characteristic peak-dip-hump profile. The two features coexist over a wide range of temperature.

Figure 2 shows the wave-vector dependence of the single-particle spectral function at the temperature $T=$ $0.7 T_{c}$ for the same coupling of Fig. 1. Following the evolution of the coherent peak across the underlying Fermi surface, one identifies the characteristic particle-hole mixing of the BCS theory, with a reflection of both particle and hole bands accompanied by a transfer of the spectral weight from negative to positive frequencies. In our theory, such BCS-like features coexist with non-BCS behaviors, namely, the occurrence of the pseudogap structure and the strong temperature dependence of the spectral weight of the coherent peak (see Fig. 4 below).

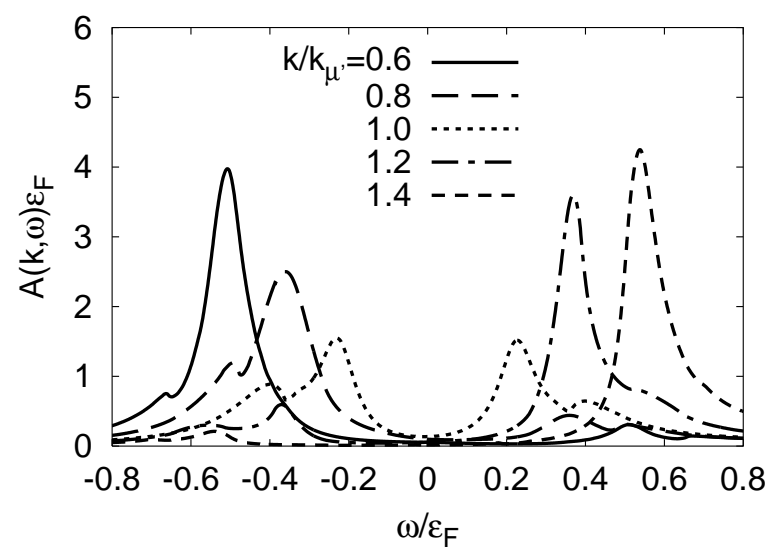

FIG. 2. Single-particle spectral function vs frequency (in units of $\left.\varepsilon_{F}\right)$ at different wave vectors about $k_{\mu^{\prime}}$, for $T=0.7 T_{c}$ and $\left(k_{F} a_{F}\right)^{-1}=-0.45$.

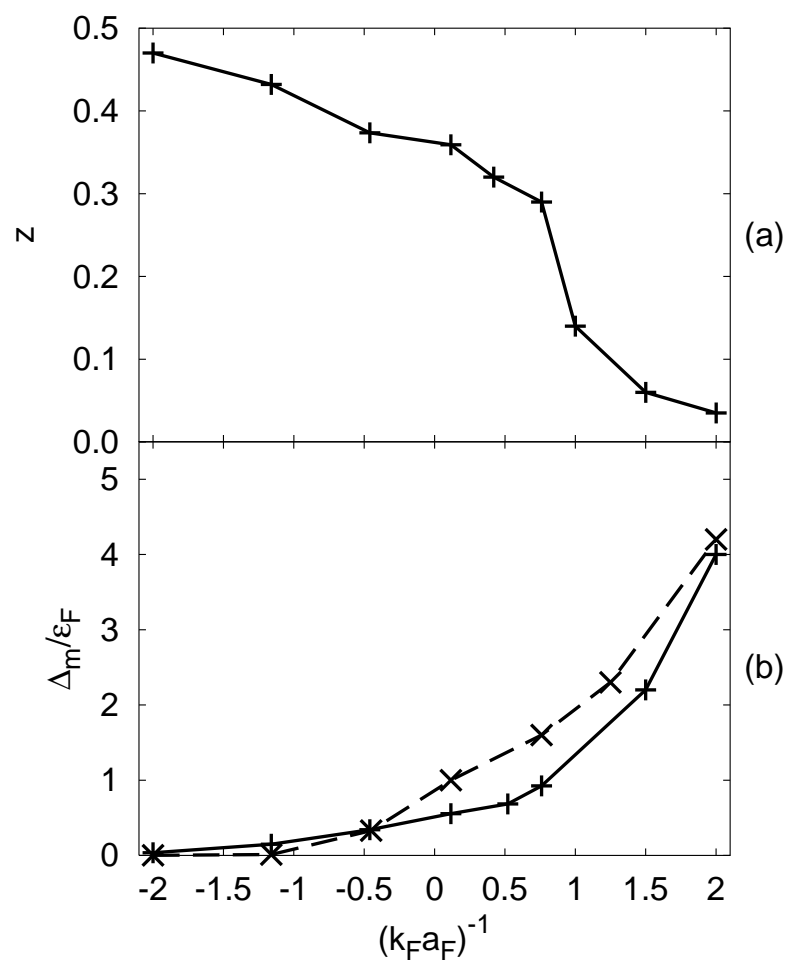

FIG. 3. (a) Weight $z$ and (b) position $\Delta_{m}$ (full line) of the coherent peak at negative frequencies vs coupling for $T=0.1 T_{c}$. In (b) the pseudogap $\Delta_{\mathrm{pg}}$ for $T=T_{c}$ (dashed line) is also shown.

Figures 3(a) and 3(b) report, respectively, the weight $z$ and position $\Delta_{\mathrm{m}}$ of the coherent peak of the singleparticle spectral function at negative frequencies vs coupling for a low temperature $\left(T=0.1 T_{c}\right)$. The peak weight $z$ saturates at the BCS value 0.5 in weak coupling, decreases markedly across the crossover region $-1 \lesssim\left(k_{F} a_{F}\right)^{-1} \lesssim+1$, and becomes negligible in strong coupling. At the same time, the peak position $\Delta_{\mathrm{m}}$ in- 
creases monotonically across the crossover region. In Fig. 3(b) we also report the pseudogap $\Delta_{\text {pg }}$ (dashed line), as identified from the position of the maximum of the spectral function at $T_{c}$. While the qualitative trend of $\Delta_{\mathrm{m}}$ and $\Delta_{\mathrm{pg}}$ vs coupling is similar, the two curves cross each other at about the intermediate-coupling value $\left(k_{F} a_{F}\right)^{-1}=-0.45$. In addition, we have verified that $\Delta_{\mathrm{m}}$ about coincides with the value of the order parameter $\Delta$ in the weak-to-intermediate coupling region.

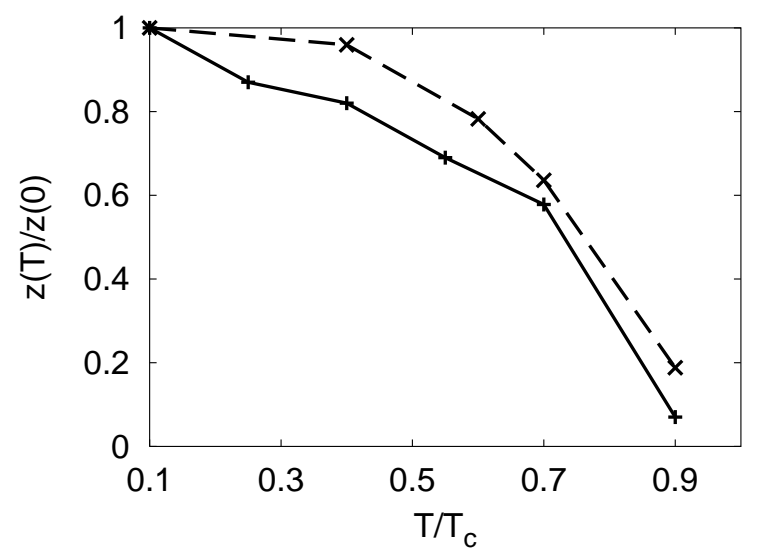

FIG. 4. Temperature dependence of the weight $z$ of the coherent peak at negative frequencies (full line) for $\left(k_{F} a_{F}\right)^{-1}=-0.45$. The superfluid density $\rho_{s}$ (dashed line) is also shown for comparison.

Figure 4 reports the dependence of the weight $z$ on temperature for the coupling value $\left(k_{F} a_{F}\right)^{-1}=-0.45$ (full line). Note the strong temperature dependence of this quantity, which vanishes at $T_{c}$. This contrasts the BCS behavior, whereby the weight of the coherent peak for negative frequencies would equal 0.5 irrespective of temperature. The temperature dependence of the superfluid density $\rho_{s}$ (calculated according to Ref. 17) is also shown in the figure (dashed line). The resemblance between these two quantities has been noted experimentally for nearly-optimally-doped cuprates ${ }^{9,10}$. We have, however, verified that this resemblance does not occur for other values of the coupling, both on the weak- and strong-coupling sides of the crossover. On the weakcoupling side of the crossover, in particular, our finding is confirmed by the BCS theory, whereby $z=0.5$ irrespective of temperature while $\rho_{s}$ decreases monotonically from $T=0$ to $T_{c}$. For this reason, no universal correspondence between the temperature dependence of $z$ and $\rho_{s}$ should be expected on physical grounds.

The results shown in the above figures refer mostly to the weak-to-intermediate coupling side of the crossover region. Our conclusions have, however, been drawn from a sistematic study of the whole crossover region from weak to strong coupling, of which Fig. 3 is an example.

All the qualitative features extracted from the above figures compare favorably with the experimental data on cuprates, as anticipated in the points (i)-(iv). This qualitative comparison rests on the assumed correspondence between the increasing of the coupling strength in the present theory and the decreasing doping level in the phase diagram of cuprate superconductors.

In conclusion, we have shown that pairing fluctuations can (at least qualitatively) account for several nontrivial features of single-particle spectra in the superconducting state. Our results specifically demonstrate that the experimental finding of two distinct features (pseudogap structure and coherent peak) in the single-particle spectral function is fully consistent with the occurrence of strong pairing fluctuations in cuprate superconductors. Competition of two distinct order parameters is therefore not required to account for the occurrence of two different energy scales in the experimental data.

We are indebted to A. Perali for discussions. Financial support from the Italian MIUR under contract COFIN 2001 Prot. 2001023848 is gratefully acknowledged.

${ }^{1}$ A. Damascelli, Z-X. Shen, and Z. Hussain, Rev. Mod. Phys. 75, 473 (2003), and references therein.

${ }^{2}$ M. Kugler et al., Phys. Rev. Lett. 86, 4911 (2001).

${ }^{3}$ D.L. Feng et al., Phys. Rev. Lett. 86, 5550 (2001); Y.-D. Chunag et al., ibid. 87, 117002 (2001); A.A. Kordyuk et al., ibid. 89, 077003 (2002).

${ }^{4}$ A.D. Gromko et al., cond-mat/0205385.

${ }^{5}$ Y. Wang et al., Science 299, 86 (2003).

${ }^{6}$ M. Eschrig and M.R. Norman, Phys. Rev. Lett. 89, 277005 (2002); G. Seibold and M. Grilli, Phys. Rev. B 63, 224505 (2001).

7 J.C. Campuzano et al., Phys. Rev. B 53, R14737 (1996).

${ }^{8}$ H. Matsui et al., Phys. Rev. Lett. 90, 217002 (2003).

${ }^{9}$ D.L. Feng et al., Science 280, 277 (2000).

${ }^{10}$ H. Ding et al., Phys. Rev. Lett. 87, 227001 (2001).

${ }^{11}$ V.M. Krasnov, Phys. Rev. B 65, 140504(R) (2002).

${ }^{12}$ V.N. Zavaritsky, cond-mat/0306081.

${ }^{13}$ P. Nozières and S. Schmitt-Rink, J. Low. Temp. Phys. 59, 195 (1985).

${ }^{14}$ A. Perali, P. Pieri, G.C. Strinati, and C. Castellani, Phys. Rev. B 66, 024510 (2002).

${ }^{15}$ P. Pieri, L. Pisani, and G.C. Strinati, unpublished.

${ }^{16}$ As it was done in Ref. 14 above $T_{c}$, in the weak-tointermediate coupling region we found it necessary to introduce the constant shift $\Sigma_{0}=\operatorname{Re} \Sigma_{11}^{R}\left(|\mathbf{k}|=k_{\mu^{\prime}}, \omega=0\right)$ in the BCS single-particle Green's functions entering Eqs. (1)-(4). This shift plays in the present context an analogous role to the Hartree shift for the negative- $U$ Hubbard model. With this shift, $A(\mathbf{k}, \omega)$ has a local minimum for $\omega=0$ at the Fermi surface crossing, where $|\mathbf{k}|$ takes the value $k_{\mu^{\prime}}=\sqrt{2 m \mu^{\prime}}$ for $\mu^{\prime}>0$ with $\mu^{\prime}=\mu-\Sigma_{0}$. The spectra for $A(\mathbf{k}, \omega)$ are shown in the text for this special values of $|\mathbf{k}|$.

${ }^{17}$ N. Andrenacci, P. Pieri, and G.C. Strinati, Phys. Rev. B 68, 144507 (2003). 